\title{
IDENTIFICAÇÃO E HIERARQUIZAÇÃO DE CRITÉRIOS E SUBCRITÉRIOS PARA SELEÇÃO DE FORNECEDORES EM UMA CONCESSIONÁRIA DE RODOVIAS UTILIZANDO O MÉTODO ANALYTIC HIERARCHY PROCESS (AHP)
}

\author{
IDENTIFICATION AND HIERARCHIZATION OF CRITERIA AND SUBCRITERIA \\ FOR SELECTING SUPPLIERS IN A ROAD CONCESSIONAIRE USING THE \\ ANALYTIC HIERARCHY PROCESS (AHP) METHOD
}

\author{
Tiago Leonesi - tleonesi@hotmail.com \\ Faculdade de Tecnologia (Fatec) - Bebedouro - SP - Brasil \\ Luis Fernando Terazzi - luis.terazzi@gmail.com \\ Faculdade de Tecnologia (Fatec) - Bebedouro - SP - Brasil
}

DOI: 10.31510/infa.v17i2.897

Data de publicação: 18/12/2020

\begin{abstract}
RESUMO
Rotineiramente as organizações se deparam com algum tipo de problema que requer uma análise de decisão, sendo muitas vezes, necessário realizar julgamentos, o que não é uma tarefa das mais fáceis. Desse modo, quando se trata de selecionar um fornecedor, são muitos os critérios a serem analisados. Assim, o objetivo dessa pesquisa é identificar e hierarquizar critérios e subcritérios para a seleção de fornecedores através do método AHP em uma concessionária de rodovias de grande porte da cidade de Bebedouro/SP. Para tanto, foi adotado uma abordagem quantitativa com modelagem matemática, sendo os dados e os julgamentos realizados por três stakeholders envolvidos no assunto. A modelagem dos julgamentos foi realizada com o auxílio do software gratuito SuperDecisions. Foram necessárias duas reuniões divididas em dois dias para apresentação do método AHP, construção da estrutura hierárquica, e julgamento dos critérios e subcritérios. A pesquisa identificou três critérios e seis subcritérios essenciais para a seleção de fornecedores na empresa alvo da pesquisa. O resultado indicou o critério Custo como o mais importante e na hierarquização e os subcritérios Preço, Cumprir prazo e Produto foram os que mais se destacaram.
\end{abstract}

Palavras-chave: Analytic Hierarchy Process. Concessionária de Rodovias. Logística

\begin{abstract}
Routinely organizations are faced with some type of problem that requires decision analysis, and it is often necessary to make judgments, which is not an easy task. Thus, when it comes to selecting a supplier, there are many criteria to be analyzed. Thus, the objective of this research is to identify and prioritize criteria and sub-criteria for the selection of suppliers through the AHP method in a large highway concessionaire in the city of Bebedouro / SP. To this end, a
\end{abstract}


quantitative approach with mathematical modeling was adopted, with data and judgments made by three stakeholders involved in the subject. The modeling of the judgments was performed with the aid of the free software SuperDecisions. It took two meetings divided into two days to present the AHP method, build the hierarchical structure, and judge the criteria and sub-criteria. The survey identified three essential criteria and six sub-criteria for selecting suppliers at the target company. The result indicated the Cost criterion as the most important and in the hierarchy, the subcriteria Price, Meet deadline and Product were the ones that stood out the most.

Keywords: Analytic Hierarchy Process. Highway Concessionaire. Logistics

\section{INTRODUÇÃO}

A eficácia na seleção de fornecedores é uma questão importante e ao mesmo tempo complexo para qualquer tipo de empresa, assim, segundo Terazzi (2020), uma grande diversidade de produtos são produzidos e disponibilizados aos consumidores, que estão mais inflexíveis e exigentes. Um julgamento incorreto, em qualquer etapa, pode levar à seleção de um ou mais fornecedores inadequados (KUMAR; PADHI; SARKAR, 2019). Assim, Kilincci e Onal (2011) e Yadav e Sharma (2016) indicam que o processo de seleção de fornecedores é indispensável para as organizações, visto que o preço das matérias-primas e componentes constitui muitas vezes o principal custo de um produto.

Para Rajesh e Malliga (2013), selecionar fornecedores é uma das decisões estratégicas indispensáveis nas empresas, desempenhando um papel significativo na gestão. De acordo com Yadav e Shama (2016), para um fornecedor se tornar efetivo é desejável que a empresa realize um procedimento ou método sistemático e eficaz para selecioná-lo levando em consideração vários parâmetros. Desse modo, Dweiri et al. (2016) inserem a seleção de fornecedores como um problema de tomada de decisão multicritérios.

Neste contexto, Kilincci e Onal (2011), Promentilla et al. (2018) indicam o método Analytic Hierarchy Process (AHP) como um dos principais procedimentos sistemático para resolver problemas de tomada de decisão de multicritérios. Sendo seu ponto forte focado na estruturação de critérios de avaliação na forma de uma hierarquia e na obtenção de preferência se stakeholdres sobre a importância dos critérios (PISHCHULOV et al., 2019).

A empresa, objeto de estudo, é uma concessionária de rodovias, localizada em Bebedouro/SP, que demonstra comprometimento nas condições de segurança e conforto oferecidas aos usuários. Segundo a Associação Brasileira de Concessionárias de Rodovias 
(ABCR) (2018) estudos comprovam o papel fundamental das rodovias tanto no presente quanto no futuro do Brasil, que possui uma necessidade iminente em solucionar os graves problemas que impedem o desenvolvimento de sua infraestrutura rodoviária. Portanto, a escolha e a priorização de critérios para seleção de fornecedores passam a ser imprescindíveis.

Desse modo, o objetivo deste artigo é, através de uma pesquisa quantitativa com modelagem matemática auxiliada através do software especialista em AHP SuperDecicions, identificar e hierarquizar critérios e subcritérios para a seleção de fornecedores em uma concessionária de rodovias de grande porte da cidade de Bebedouro/SP. Antes, entretanto, de efetuar este estudo prático (cuja metodologia sé caracterizados na seção 3 e os resultados na seção 4), a seção 2, que se inicia a seguir, apresenta uma revisão da literatura que contempla os assuntos que sustentam o estudo que aqui se propõe.

\section{FUNDAMENTAÇÃO TEÓRICA}

\subsection{Seleção de Fornecedores}

Segundo Macohin (2012), as empresas que buscam a perfeição operacional devem introduzir programas para o desenvolvimento de seus fornecedores com o propósito de garantir uma entrega eficiente, reduzir de custos, estabelecer parcerias, flexibilizar e agilizar os processos de fornecimento de suprimentos ou serviços. Desse modo, Bowersox et al. (2014) relatam que uma inclusão operacional maior pode ocorrer quando compradores e fornecedores atuam em conjunto para identificar os processos necessários para reduzir o tempo do pedido e extinguir erros de comunicação. Portanto, a integração pode assumir diversas formas como, por exemplo, o comprador permitir ao fornecedor o acesso das informações de vendas e pedidos, concedendo a ele conhecimento amplo dos seus produtos.

Kumar, Padhi e Sarkar (2019) ressaltam que cada indústria é diferente entre si e por isso devem observar pontualmente os critérios de seleção de seus fornecedores. A opinião dos especialistas envolvidos é tratada como uma base relevante. Desse modo, Taherdoost e Brard (2019) indicam que o principal objetivo ao realizar um processo de seleção de fornecedores é: diminuir o risco da compra, aumentar o valor geral para o comprador e aproximar-se e criar relações a longo prazo com o fornecedor. 
Ho, Xu e Dey (2010) indicaram em sua pesquisa que os critérios mais populares para seleção de fornecedores são: qualidade, prazo de entrega, preço/custo, capacidade de produção, serviço, tecnologia, pesquisa e desenvolvimento, flexibilidade, reputação, relacionamento, risco e segurança e meio ambiente. Indicando que o desempenho de fornecedores considerados como potenciais é qualificado de acordo com vários critérios, ao invés de se considerar apenas um fator.

\subsection{AHP}

De acordo com Silva (2018 apud SANTOS, 2009), o AHP foi a metodologia pioneira das decisões com múltiplos critérios de escolha. O AHP é considerado um dos primeiros métodos criados para ajudar na tomada de decisão baseado em múltiplos critérios, permitindo aos stakeholders encontrar uma melhor alternativa para a solução de algum problema dentro de uma organização. Foi introduzido pela primeira vez por Thomas Lorie Saaty em 1980, propondo que os tomadores de decisões forneçam julgamentos sobre a importância relativa de cada critério e, em seguida, especifiquem uma preferência para cada alternativa de decisão (LABIB, 2014). Neste contexto, Fu (2019) identifica que o aspecto do AHP é escolher a melhor alternativa através de julgamentos partindo de um conjunto de escolhas competitivas e avaliá-las à luz de um conjunto de critérios. Portanto, o primeiro passo para aplicar o AHP é definir o objetivo e estabelecer rigorosamente os critérios de seleção. De acordo com Dweiri et al. (2016), o segundo passo é, se necessário, identificar e criar subcritérios que melhor descrevamos critérios definidos para avaliar um conjunto de alternativas,

Saaty (1990) define que primeiramente o problema deve ser traduzido numa hierarquia de prioridades, conforme Figura 01, dividido e estruturado com as suas características, para em seguida ser colocado em prática no modelo AHP. Desse modo, de acordo com Saaty (2008), para tomar uma decisão de maneira organizada são necessárias quatro etapas: (1) definir o problema; (2) criar e estruturar uma hierarquia de decisão com os seguintes pontos: objetivo, critérios, subcritérios e alternativas caso seja necessário; (3) construir as matrizes de comparação pareadas identificando o vetor prioridade e (4) usar as prioridades conquistadas nas comparações para ponderar as prioridades no nível imediato inferior. 
Figura 01 - Hierarquia de decisão típica do AHP

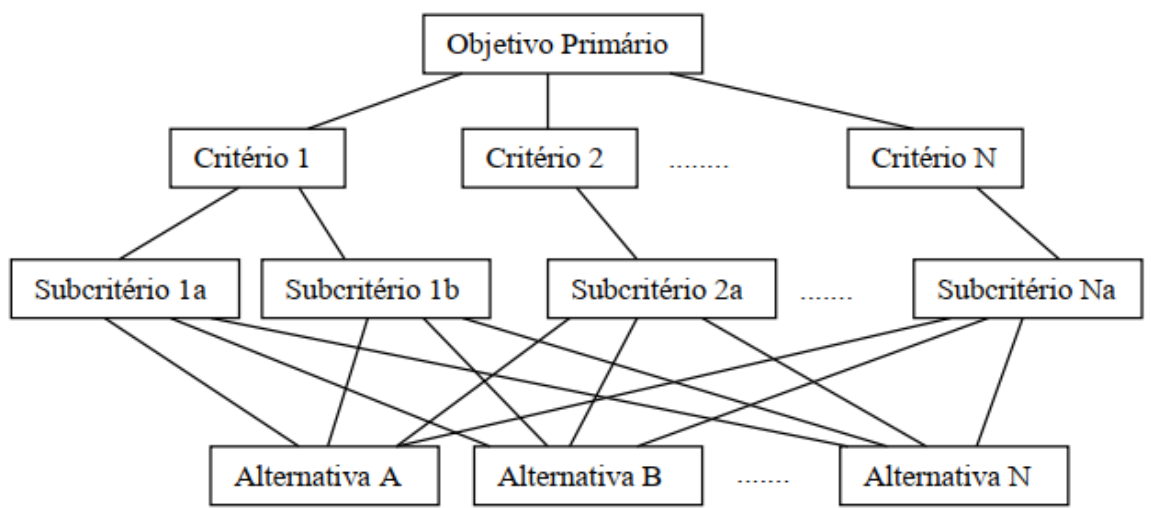

Fonte: adaptado de Labib (2014).

Power (2014) define o AHP como uma ferramenta para tomada de decisões que engloba a estruturação de multicritérios de escolha com base em numa hierarquia. $\mathrm{O}$ método AHP é direcionado para avaliar a importância relativa dos critérios, comparando cada alternativa com seu subcritério especifico. Assim, pode ser criado um ranking com as principais alternativas selecionadas. Saaty (2008) preconiza que para realizar comparações, é primordial uma escala de números que indiquem quanta vez mais importante é um elemento em relação ao outro. O Quadro 01 exibe esta escala.

\begin{tabular}{|c|l|l|}
\hline $\begin{array}{c}\text { Intensidade de } \\
\text { Importância }\end{array}$ & \multicolumn{1}{|c|}{ Definição } & \multicolumn{1}{c|}{ Explicação } \\
\hline 1 & Importância igual & $\begin{array}{l}\text { Duas atividades contribuem igualmente para o } \\
\text { objetivo. }\end{array}$ \\
\hline 3 & Importância moderada & $\begin{array}{l}\text { Experiência e julgamento favorecem ligeiramente } \\
\text { uma atividade em detrimento de outra. }\end{array}$ \\
\hline 5 & Forte importância & $\begin{array}{l}\text { Experiência e julgamento favorecem fortemente uma } \\
\text { atividade sobre outra. }\end{array}$ \\
\hline 7 & $\begin{array}{l}\text { Muito forte ou } \\
\text { importância demonstrada }\end{array}$ & $\begin{array}{l}\text { Uma atividade é favorecida muito fortemente em } \\
\text { detrimento de outra. }\end{array}$ \\
\hline 9 & Extrema importância & $\begin{array}{l}\text { A evidência que favorece uma atividade em detrimento } \\
\text { de outra é da mais alta ordem possível de afirmação. }\end{array}$ \\
\hline $2,4,6,8$ & Valores intermediários & Utilizados como valores de consenso entre as opiniões. \\
\hline
\end{tabular}
Fonte: adaptado de Saaty (2008).

Segundo Carvalho, Kruk e Belderrain (2016) a produção de um julgamento de valor é alicerce do método AHP, contudo, pode-se ocorrer avaliações inconsistentes em alguns casos. Considerando esta possibilidade, o método propõe procedimentos que permitem avaliar o Índice de Consistência (IC) dos julgamentos. Saaty (1990) indica que o IC deve ser inferior a 
$10 \%$ para matrizes de ordem $\mathrm{n} \geq 3$. Para inconsistências maiores, sugere uma revisão dos julgamentos por parte dos stakeholders.

\subsection{Importância do AHP na seleção de fornecedores}

Várias técnicas são sugeridas para resolver o problema de seleção de fornecedores (FU, 2019). Cada vez mais as empresas depositam confiança nos seus fornecedores para melhorar a qualidade dos seus produtos e serviços, reduzir custos ou focar numa parte específica das suas operações (GOVINDAN et al., 2015). Vários métodos de decisão multicritério foram propostas para a seleção de fornecedores, sendo o AHP, uma das metodologias mais robustas utilizadas (HO; XU; DEY, 2010). Segundo Ho e Ma (2017) e Kumar, Padhi e Sarkar (2019), o AHP é o método mais amplamente utilizado para seleção de fornecedores.

O uso do AHP na seleção do fornecedor dá ao tomador de decisão a confiança, a consistência e a robustez em uma tomada de decisão (DWEIRI et al., 2016). De acordo com $\mathrm{Fu}$ (2019), o primeiro passo para usar o AHP na seleção de fornecedores é definir, preferencialmente com especialistas da área, os critérios primários (mais próximos ao objetivo), pois servirão para guiar a sequência da pesquisa. Em seguida, para Kumar, Padhi e Sarkar (2019), a classificação do peso, em comparações pareadas, de cada critério é uma questão importante e que servirá de alicerce para tomar uma decisão. Desse modo, segundo Deng et al. (2014), a seleção de fornecedores e a aplicação do AHP irão depender muito da avaliação e do conhecimento dos especialistas envolvidos no processo.

\section{PROCEDIMENTOS METODOLÓGICOS}

O presente artigo é enquadrado em uma abordagem quantitativa que utiliza a técnica da modelagem matemática auxiliada pelo software gratuito especialista em AHP SuperDecisons. Segundo Turrioni e Mello (2012) a abordagem qualitativa é uma conexão entre o mundo real e o sujeito, onde a principal ferramenta é o pesquisador e a coleta de dados, e os processos e seus significados são fundamentais para o tipo de abordagem. $\mathrm{O}$ estudo é realizado em uma Concessionária de Rodovias de grande porte que atua no ramo de prestação de serviço. Está localizada em Bebedouro/SP e administra um trecho de rodovias de 
aproximadamente 155 quilômetros, oferecendo aos seus usuários serviços como: atendimento pré-hospitalar, guincho, socorro mecânico, reformas e obras necessárias. Vale a pena destacar que o pesquisador é colaborador da empresa, facilitando o levantamento dos dados da pesquisa.

Dessa maneira, constatado o problema para identificar e hierarquizar critérios para seleção de fornecedores, foi desenvolvido um procedimento para estruturar a metodologia. Primeiramente, foi realizado uma pesquisa em livros, artigos e sites para alicerçar e criar uma fronteira referente ao contexto da pesquisa. Em um segundo momento, foi realizada a escolha de três stakeholders, sendo um Gerente de Operações, um Comprador e um Diretor de Obras e Engenharia, com 13, 8 e 17 anos no cargo respectivamente.

Desse modo, foi repassado todo o conteúdo atribuído a este estudo aos entrevistados, com uma ênfase na teoria pertinente ao AHP. Com todos presentes em uma sala de reunião, respeitando os procedimentos higiênicos sanitários da organização, os dados, em consenso de opiniões, foram coletados, julgados e registrados no software SuperDecisions. Vale a pena destacar que os julgamentos foram realizados em consenso pelos stakeholders e que o critério de aprovação foi o IC abaixo de 10\%, conforme proposto por Saaty (1990). Essa modelagem foi adotada para que os stakeholders, idealizassem os julgamentos e os vetores prioridades, e no caso de alguma divergência na escolha, poderiam em consenso adequá-las no momento do

julgamento. As reuniões foram divididas em dois dias, com uma média de 3 horas cada. Após a conclusão dos julgamentos, os resultados finais foram apresentados para validação.

\section{RESULTADOS E DISCUSSÃO}

Para a execução do estudo, de maneira consensual, primeiramente os stakeholders caracterizaram os critérios para seleção de fornecedores que se identificavam com o objetivo da organização, sendo: Custo, Qualidade e Prazo. Desse modo, para criar a hierarquia da pesquisa, os subcritérios de seleção de fornecedores, também foram identificados de modo consensual, e alocados em seus respectivos critérios pelos stakeholders, ficando classificados conforme Figura 02. 
Figura 02 - Hierarquia e divisão para seleção de fornecedores.

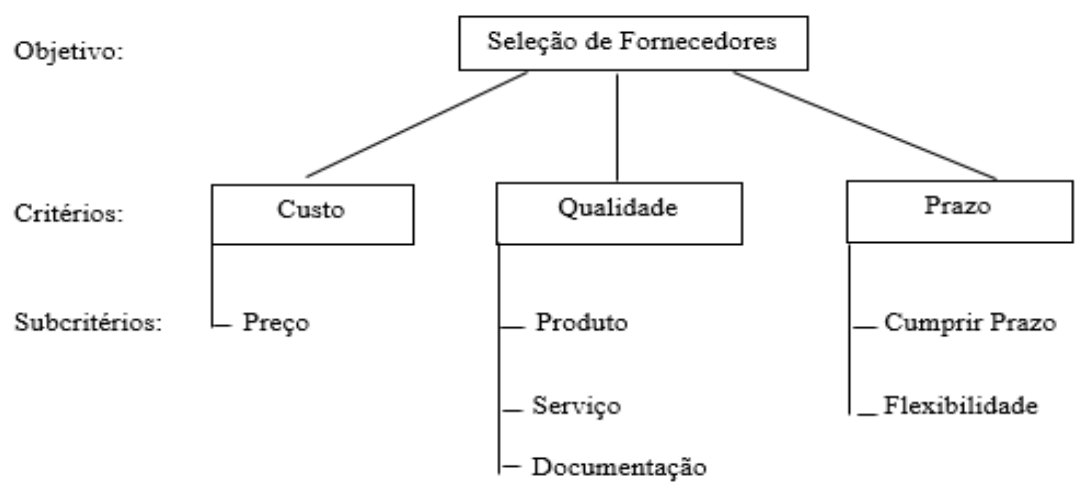

Fonte: elaborado pelos autores (2020).

Após a etapa de identificação dos critérios e subcritérios, em consenso com os stakeholders, foram realizadas as comparações pareadas primeiramente dos critérios, conforme Tabela 01. Destaca-se que todos os IC foram obtidos através do software SuperDecions.

Tabela 01 - Comparação dos julgamentos dos critérios de seleção.

\begin{tabular}{|c|c|c|c|}
\hline Critérios Logísticos & Custo & Qualidade & Prazo \\
\hline Custo & 1,0000 & 1,2000 & 1,4000 \\
\hline Qualidade & 0,8333 & 1,0000 & 1,0000 \\
\hline Prazo & 0,7143 & 1,0000 & 1,0000 \\
\hline $\mathrm{IC}=0,260 \%$ & 2,5476 & 3,2000 & 3,4000 \\
\hline
\end{tabular}

Fonte: elaborado pelos autores (2020).

$\mathrm{Na}$ Tabela 02, é realizado a normalização e a descoberta do vetor prioridade do julgamento dos critérios. Vale a pena ressaltar que, todos os valores identificados no vetor prioridade são apresentados e analisados em porcentagem.

Tabela 02 - Normalização e vetor prioridade da matriz de julgamento dos critérios.

\begin{tabular}{lcccc}
\hline Critérios Logísticos & Custo & Qualidade & Prazo & Vetor Prioridade \\
\hline Custo & 0,3925 & 0,3750 & 0,4118 & $\mathbf{0 , 3 9 3 1}$ \\
Qualidade & 0,3271 & 0,3125 & 0,2941 & $\mathbf{0 , 3 1 1 2}$ \\
Prazo & 0,2804 & 0,3125 & 0,2941 & $\mathbf{0 , 2 9 5 7}$ \\
\hline
\end{tabular}

Fonte: elaborado pelos autores (2020).

Após as informações coletadas, obteve-se o resultado do vetor prioridade dos critérios, conforme Tabela 02. Observa-se que os stakeholders preferem: 1 - Custo - 39,31\%; 2 Qualidade - 31,12\%; e 3 - Prazo - 29,57\%. Após uma análise de sensibilidade, os Gerentes de 
Operação e o Diretor de Obras e Engenharia indicaram que resultado demonstra que a empresa prioriza o custo, com bastante ênfase em qualidade, pois acreditam que nos dias atuais esses critérios são essenciais para o sucesso na seleção de fornecedores. Dweiri et al. (2016) concluem que as organizações buscam atender à demanda do cliente com economia e qualidade. A Tabela 03 indica os julgamentos realizados em relação aos subcritérios da Qualidade.

Tabela 03 - Matriz de julgamento do subcritérios de Qualidade.

\begin{tabular}{|c|c|c|c|c|}
\hline Subcritérios & & Produto & Serviço & Documentação \\
\hline Produto & & 1,0000 & 1,0000 & 1,5000 \\
\hline Serviço & & 1,0000 & 1,0000 & 1,4000 \\
\hline Documentação & & 0,6667 & 0,7143 & 1,0000 \\
\hline $\mathrm{IC}=0,051 \%$ & Total & 2,6667 & 2,7143 & 3,9000 \\
\hline
\end{tabular}

A Tabela 04 indica vetor prioridade dos subcritérios de Qualidade, o julgamento foi considerado válido, pois o IC encontrado no julgamento foi de $0,051 \%$.

Tabela 04 - Normalização e vetor prioridade da matriz do subcritérios de Qualidade.

\begin{tabular}{lcccc}
\hline Subcritérios & Produto & Serviço & Documentação & Vetor Prioridade \\
\hline Produto & 0,3750 & 0,3684 & 0,3846 & $\mathbf{0 , 3 7 6 0}$ \\
Serviço & 0,3750 & 0,3684 & 0,3590 & $\mathbf{0 , 3 6 7 5}$ \\
Documentação & 0,2500 & 0,2632 & 0,2564 & $\mathbf{0 , 2 5 6 5}$ \\
\hline
\end{tabular}

Fonte: elaborado pelos autores $(\mathbf{2 0 2 0})$.

No contexto dos subcritérios de Qualidade, observa-se que a qualidade referente ao Produto se destaca com $37,60 \%$ das preferências ao selecionar um fornecedor. O subcritério Serviço se posiciona próximo com $36,75 \%$ das preferências. Na análise dos stakeholders são fundamentais na escolha de fornecedores, pois acreditam que com esses subcritérios a empresa procura diminuir o retrabalho e também o desperdício.

A Tabela 05 demonstra o julgamento dos stakeholders em relação aos subcritérios em questão do critério Prazo.

Tabela 05 - Matriz de julgamento dos subcritérios de Prazo.

\begin{tabular}{|c|c|c|}
\hline Critérios Logísticos & Cumprir Prazo & Flexibilidade \\
\hline Cumprir Prazo & 1,0000 & 2,0000 \\
\hline Flexibilidade & 0,5000 & 1,0000 \\
\hline $\mathrm{IC}=0,000 \%$ & 1,5000 & 3,0000 \\
\hline
\end{tabular}


A Tabela 06 indica o vetor prioridade dos subcritérios relacionados com o Prazo.

Tabela 06 - Normalização e vetor prioridade da matriz do subcritérios de Prazo.

\begin{tabular}{lccc}
\hline Critérios Logísticos & Cumprir Prazo & Flexibilidade & Vetor Prioridade \\
\hline Cumprir Prazo & 0,6667 & 0,6667 & $\mathbf{0 , 6 6 6 7}$ \\
Flexibilidade & 0,3333 & 0,3333 & $\mathbf{0 , 3 3 3 3}$
\end{tabular}

Fonte: elaborado pelos autores (2020).

Desse modo, o subcritério para seleção de fornecedores Cumprir Prazo com 66,67\% é o mais importante segundo a análise dos stakeholders, pois garante que seja feito um planejamento eficaz no cumprimento de um serviço ou entrega de produto na data correta.

Neste contexto, depois de todos os critérios e subcritérios serem definidos individualmente foi criado uma hierarquização para uma classificação geral de importância (vetor prioridade do critério $\mathrm{x}$ vetor prioridade da seleção de fornecedores). O Quadro 02 apresenta, com informações obtidas na pesquisa, o ranking com os subcritérios mais importante para a seleção de fornecedores da Concessionária de rodovias.

Quadro 02 - Ranking dos subcritérios mais importantes para a seleção de um fornecedor.

\begin{tabular}{|c|c|c|c|c|c|}
\hline \multirow{2}{*}{$\begin{array}{l}\text { Critérios para Seleção de } \\
\text { Fornecedores } \\
\text { Subcritérios na seleção de } \\
\text { fornecedores }\end{array}$} & Custo & Qualidade & Prazo & \multirow[t]{2}{*}{$\begin{array}{c}\text { Vetor } \\
\text { Prioridade Total }\end{array}$} & \multirow[t]{2}{*}{ Ranking } \\
\hline & 0,3931 & 0,3112 & 0,2957 & & \\
\hline Preço & 1,0000 & & & 0,3931 & $1^{\circ}$ \\
\hline Produto & & 0,3760 & & 0,1170 & $3^{\circ}$ \\
\hline Serviço & & 0,3675 & & 0,1144 & $4^{\circ}$ \\
\hline Documentação & & 0,2565 & & 0,0798 & $6^{\circ}$ \\
\hline Cumprir Prazo & & & 0,6667 & 0,1971 & $2^{\circ}$ \\
\hline Flexibilidade & & & 0,3333 & 0,0986 & $5^{\circ}$ \\
\hline
\end{tabular}

Fonte: elaborado pelos autores (2020).

Assim, segundo as informações do Quadro 02, obtém-se que os subcritérios Preço com 39,30\%, Cumprir Prazo com 19,33\% e Produto 11,70\% são os três quesitos com o maior destaque na organização ao selecionar um fornecedor. Segundo os stakeholders, é importante essa hierarquização pois estrutura-se um direcionamento para precisão da seleção de fornecedores. Neste contexto, Terazzi (2020) indica que com a criação uma hierarquia através do AHP fica evidente os requisitos necessários para a aderência de todos os envolvidos e um direcionamento correto da gestão. Destaca-se que todos os entrevistados indicaram que todos 
os critérios e subcritérios são importantes para a organização. Vale a pena destacar que, neste trabalho, todos os resultados foram modelados no software de AHP SuperDecisions.

\section{CONCLUSÃO}

É notório perceber uma evolução na administração das organizações, assim sendo, a escolha dos requisitos para selecionar um fornecedor deve ser analisada de acordo com suas necessidades pontuais. Diante deste cenário, a organização alvo da pesquisa, identificou e hierarquizou através de seus stakeholders os critérios e subcritérios mais importantes para a seleção de fornecedores utilizando a metodologia AHP.

Portanto, identifica-se que o objetivo proposto neste artigo foi atingido, sendo a metodologia AHP muito útil para auxiliar as tomadas de decisão complexas de serem decididas. Através do estudo realizado, a partir metodologia AHP, fica claro e simples de identificar o que a organização preza pelo subcritério Preço, seguido do requisito Cumprir prazo e posteriormente do Produto. Notou-se que a organização busca um preço acessível, sem deixar de dar a devida importância no prazo e também na qualidade do produto, pois acredita que com esses conceitos a empresa consegue selecionar o fornecedor ideal para serviços apropriado.

\section{REFERÊNCIAS}

ASSOCIAÇÃO BRASILEIRA DE CONCESSIONÁRIAS DE RODOVIAS (ABCR). Novos caminhos para concessões de rodovias no Brasil. 2018. Disponível em <

https://abcr.org.br/Library/novoscaminhos/abcr_relatorio_novos_caminhos.pdf $>$. Acesso em: 30 ago. 2020.

BOWERSOX, D. J.; et al. Gestão Logística da Cadeia de Suprimentos. Porto Alegre: Editora AMGH, 2014.

CARVALHO, R.G.; KRUK, N.S.; BELDERRAIN, M.C.N. Aplicação do método de análise hierárquica para seleção de sistemas de separação água/óleo em aeroportos para diferentes cenários. Blucher Marine Engineering Proceedings, v.2, n.1, p. 64-75, 2016.

DENG, X.; HU, Y.; DENG, Y.; MAHADEVAN, S. Supplier selection using AHP methodology extended by D numbers. Expert Systems with Applications, v.41, n. 1, p.156167, 2014. 
DWEIRI, F.; KUMAR, S.; KHAN, S.A.; JAIN, V. Designing an integrated AHP based decision support system for supplier selection in automotive industry. Expert Systems With Applications, v.62, p.273-283, 2016.

FU, Y.K. An integrated approach to catering supplier selection using AHP-ARASMCGP methodology. Journal of Air Transport Management, v. 75, p. 164-169, 2019.

GOVINDAN, K.; et al. Multi criteria decision making approaches for green supplier evaluation and selection: a literature review. Journal of Cleaner Production, v.98, p.66-83, 2015 .

HO, W.; XU, X.; DEY, P.K. Multi-criteria decision making approaches for supplier evaluation and selection: A literature review. European Journal of Operational Research, v.202, n.1, p. 16-24, 2010.

KILINCCI, O.; ONAL, S. A. Fuzzy AHP approach for supplier selection in a washing machine company. Expert Systems with Applications, v.38, n. 8, p.9656-9664, 2011.

KUMAR, R.; PADHI, S. S.; SARKAR, A. Supplier selection of an Indian heavy locomotive manufacturer: An integrated approach using Taguchi loss function, TOPSIS, and AHP. IIMB Management Review, v.31, n.1, p.7890, 2019.

LABIB, A. Learning from Failures: Decision Analysis of Major Disasters. ButterworthHeinemann, 2014.

MACOHIN, G. A. Compras, Contratações e Terceirizações. Curitiba: Editora IESDE Brasil S.A, 2012.

PISHCHULOV, G.; TRAUTRIMS, A.; CHESNEY, T.; GOLD, S.; SCHWAB, L. The Voting Analytic Hierarchy Process revisited: A revised method with application to sustainable supplier selection. International Journal of Production Economics, v. 211, p.166-179, 2019.

POWER, D. J. Decision Support Systems Glossary, DSSResources. 2014. Disponível em $<$ https:// https://dssresources.com/glossary>. Acesso em: 16 set. 2020.

PROMENTILLA, M.A.B.; AVISO, K.B.; LUCAS, R.I.G.; RAZONA, L.F.; TANA, R.R. Teaching Analytic Hierarchy Process (AHP) in undergraduate chemical engineering courses. Education for Chemical Engineers, v. 23, p. 34-41, 2018.

RAJESH, G.; MALLIGA, P. Supplier Selection based on AHP QFD Methodology. Procedia Engineering, v.64, p.1283-1292, 2013.

SAATY, T.L. How to make a decision: The analytic hierarchy process. European Journal of Operational Research, v.48, n.1, p.9-26, 1990.

SAATY, T.L. Decision making with the analytic hierarchy process. Int. J. Services Sciences, v. 1, n. 1, p. $83-98,2008$. 
SILVA, H. A. Estudos sobre gestão de operações em pequenas e médias empresas. Curitiba: Editora Appris, 2018.

TAHERDOOST, H.; BRARD, A. Analyzing the Process of Supplier Selection Criteria and Methods. Procedia Manufacturing, v. 32, p. 1024-1034, 2019.

TERAZZI, L.F. Proposta de um procedimento para mensuração do desempenho logístico a partir de uma empresa do ramo moveleiro de médio porte. $2020.110 \mathrm{p}$. Dissertação (Mestrado em Engenharia de Produção) - UNIARA, Araraquara, 2020.

TURRIONI, J. B.; MELLO, C. H. Metodologia de pesquisa em Engenharia de Produção: estratégias, métodos e técnicas para condução de pesquisas quantitativas e qualitativas. Itajubá: Unifei, 2012.

YADAV, V.; SHARMA, M.K. Multicriteria supplier selection model using the Analytic Hierarchy Process approach. Journal of Modelling in Management, v.11, n.1, 2016. 